\title{
ERGODICITY OF CERTAIN CYLINDER FLOWS
}

\author{
by \\ David Alan Pask
}

Thesis submitted for the degree of Doctor of Philosophy at the University of Warwick Mathematics Institute. June 1989. 


\section{SUMMARY}

In this thesis we study two types of cylinder flow, the first given by the irrational rotation, and the second arising from the von Neumann-Kakutani adding machine. We give classes of functions which define cocycles for these transformations, and determine their cohomological properties.

In Chapter 1 we give some basic definitions and results which will be used later on in this thesis.

In Chapter 2, for the irrational rotation, we give for a family of classes of functions, a family of classes of irrational numbers which give an ergodic cylinder flow (skew-product). We define, for each $k \geq 1$, a class of functions $f$ which are $k$ times differentiable a.e., with:

(i) $\int f d \mu=0$ and $f^{(k-2)}$ is continuous (if $k>1$ ),

(ii) $f^{(k-1)}$ is piecewise continuous also with zero integral,

(iii) $f^{(k)}$ is Riemann integrable with non-zero integral.

We show that the skew product extension of the irrational rotation on the circle, $\mathbf{T}$ by this class of functions is ergodic for a class of irrational numbers $\mathcal{S}_{k}$. The family of irrational numbers $\mathcal{S}_{k}$, for $k \geq 1$, is defined to be those irrational numbers whose partial quotients satisfy:

$$
\limsup \frac{a_{n+1}}{q_{n}^{k-1}}>0 .
$$

This extends a result by P. Hellekalek and G. Larcher done in [HL1] and [HL2], and also generalises the result by the author given in $[\mathrm{P}]$.

In Chapter 3 we show that for the class of functions given by $k=1$ above, we have an ergodic skew product for all adding machine transformations, this again extends work done by P. Hellekalek and G. Larcher in [HL3]. We observe that this result may not be extended, using the methods of Chapter 2, to $k>1$ and obtain ergodicity for a class of adding machine transformations. We also give a class of functions which define cocycles which are always coboundaries for a certain class of adding machine. This is analogous to the work of M. Herman [He], who has shown that the same class of functions, for the irrational rotation, define cocycles which are coboundaries for almost all irrational numbers. 


\section{Contents}

$\begin{array}{ll}\text { INTRODUCTION } & 4\end{array}$

$\begin{array}{lll}1 & \text { BACKGROUND } & 7\end{array}$

1.1 Cocycles, Essential Values . . . . . . . . . . . . . . 7

1.2 Diophantine Approximation . . . . . . . . . . . . . 8

1.3 The Adding Machine . . . . . . . . . . . . . . . . . . . . . . . 10

1.4 Skew Products . . . . . . . . . . . . . . . . 11

1.5 Assorted Results . . . . . . . . . . . . . . . 11

2 THE IRRATIONAL ROTATION 14

2.1 Introduction . . . . . . . . . . . . . . . . 14

2.2 Conditions for ergodicity . . . . . . . . . . . . . . . . . 14

3 THE ADDING MACHINE 30

3.1 Introduction . . . . . . . . . . . . . . 30

3.2 Conditions for ergodicity . . . . . . . . . . . . . . . 30

3.3 Coboundaries for the adding machine . . . . . . . . 35

$\begin{array}{lr}\text { REFERENCES } & 38\end{array}$ 


\section{Acknowledgement.}

I am grateful to my supervisor Professor Klaus Schmidt for his advice, encouragement and especially for the help given in the preparation of the paper $[\mathrm{P}]$ and this thesis. I would also like to thank Professor Peter Walters for some fruitful conversations. My thanks also go to everyone, too numerous to mention, who have made my time at the Mathematics Department so enjoyable. I acknowledge the support of SERC grant number 85318881, and the Warwick Undergraduate supervision system.

\section{Declaration.}

The material in Chapter 2 and some of Chapter 3, has been adapted from a proof given by the author in a paper submitted to the Israel Journal of Mathematics in January 1989, see [P].

\section{Dedication.}

This thesis is dedicated to my parents, and to Jacqueline Ramagge, for their constant support and enthusiasm. 


\section{INTRODUCTION}

The subject of this thesis is cylinder flows defined by the irrational rotation and the von Neumann-Kakutani adding machine transformation on the circle $\mathbf{T}$. These are two types of skew products (or cylinder cascades) which have been studied by various authors. The skew product over the irrational rotation will be dealt with in Chapter 2, and the skew product over the adding machine will be studied in Chapter 3. Chapter 1 forms a basic background of details needed in these later Chapters.

The conditions for ergodicity, given in Chapter 2, are on the first $k$ derivatives $k \geq 1$, of a continuous function (or piecewise absolutely continuous in the basic, $k=1$ case) whose integral is zero. This is important when seen in the context of results of L. Baggett [B] and M. Herman [He], when if the function is smooth, then it is a coboundary for certain irrational numbers.

We also note the work of I. Oren [O], who has shown that for rotations by any irrational number $\alpha$, step functions of the form $\chi_{[0, \beta]}-\beta$ give rise to ergodic skew products if and only if $\beta$ is irrational or $1, \alpha$ and $\beta$ are linearly independent over the rationals. Also K. Peterson [Pe1] has shown that such step functions define cocycles which are coboundaries if and only if $\beta \in \mathbf{Z} \alpha$. Thus it suffices for us to study continuous functions, and properties of their derivatives which give ergodic skew products for certain irrational numbers.

For the basic $k=1$ case the situation becomes most clear: P. Hellekalek and G. Larcher [HL1], have demonstrated ergodicity for continuously differentiable functions with a single discontinuity, for all irrationals. On the other hand, a result of M. Herman [He] gives us that a $C^{1}$ function defines a coboundary for a certain class of irrational number, so even though the functions in [HL1] are close to being $C^{1}$, the functions are certainly not coboundaries. In the paper $[\mathrm{P}]$ we give a class of functions which are piecewise absolutely continuous with a derivative which is Riemann integrable with non-zero integral, and demonstrate ergodicity for all irrationals. Also, M. Herman [He] has shown that if $f$ is absolutely continuous with a derivative $f^{\prime}$ of bounded variation, then $f$ is a coboundary for almost all irrationals. So the functions given in $[\mathrm{P}]$ are close, in some sense to both these classes of functions which M. Herman has shown to be coboundaries, however the discontinuities which give rise to a non-zero integral for the derivative, gives us ergodicity for all irrationals.

We must also compare the result for $k=1$, given in $[\mathrm{P}]$ with that of 
L. Baggett and K. Merrill $[\mathrm{BM}]$. They have shown that if $f$ is piecewise absolutely continuous with $f^{\prime} \in L^{2}$ and $\int f^{\prime} d \mu=0$, then $f$ is a coboundary for those irrational numbers with bounded partial quotients. So again, we have a fine distinction between functions which have different cohomological properties.

The results given here are extensions of the work done by P. Hellekalek and G. Larcher [HL2] who give a result for functions $f:[0,1] \rightarrow \mathbf{R}$ which are $k$ times continuously differentiable $k \geq 2$, whose derivatives satisfy:

(i) $f^{(j)}(0)=f^{(j)}(1)$, for $j=0, \ldots, k-2$,

(ii) $f^{(k-1)}(0) \neq f^{(k-1)}(1)$, and $\int_{0}^{1} f d \mu=0$.

They give a class of irrational number which guarantee ergodicity for this class of functions, for all $n$ these numbers satisfy

$$
\left\|q_{n} \alpha\right\|<\frac{1}{q_{n}^{2 k+1}} .
$$

Here we weaken the conditions on the final derivatives, we only require that:

(i) $\int f d \mu=0$ and $f^{(k-2)}$ is continuous (if $k>1$ ),

(ii) $f^{(k-1)}$ is piecewise continuous with zero integral,

(iii) $f^{(k)}$ is Riemann integrable, with non-zero integral.

We prove ergodicity for a wider class of irrational number $\mathcal{S}_{k}$ for $k \geq 1$, these irrational numbers are those whose partial quotients have the property that $\lim \sup \frac{a_{n+1}}{q_{n}^{k-1}}>0$, that is, there is a positive constant $K$ such that, for infinitely many $n$ we have that

$$
\left\|q_{n} \alpha\right\|<\frac{K}{q_{n}^{k}} .
$$

Again these results must also be seen in the light of the work of M. Herman $[\mathrm{He}]$, who has demonstrated that $C^{k}$ functions are coboundaries for a class of irrational number which satisfy 


$$
\left\|q_{n} \alpha\right\| \geq \frac{D}{q_{n}^{r}}
$$

for all $n$, where $r$ is any number such that $r<k$. Thus, as above, despite the fact that our functions are close, in some sense, to $C^{k}$ functions, we get an ergodic skew product for a class of irrational numbers.

The conditions for ergodicity given in Chapter 3, correspond to those given in Chapter 2, for $k=1$. Results on the ergodicity of step functions of zero integral over the adding machine have been given by P. Hellekalek [H2]. For example, in [H2, Theorem 2] it is shown that step functions of the form $\chi_{[0, \beta)}-\beta$ give rise to ergodic skew products if and only if $\beta$ is irrational, or strictly non-q-adic. Also [H2, Theorem 3] gives us results for more general step functions. Also from [H1], we know that such step functions define cocycles which are coboundaries if and only if $\beta$ is $\mathbf{q}$-adic.

Firstly, for the basic $k=1$ case, we demonstrate ergodicity of the skew product for all adding machine transformations. This extends the work of P. Hellekalek and G. Larcher [HL3] who use a Lipschitz derivative condition for functions with a single, non-zero jump on a particular type of adding machine. We then observe that, using the methods of Chapter 2 does not give us an extension of this result for $k>1$.

In the latter part of this Chapter we show that functions with zero integral, and have a derivative of bounded variation, define cocycles which are always coboundaries for a certain class of adding machine transformation. This is analogous to the result by M. Herman [He], who has shown that this same class of functions are also coboundaries for almost all irrationals.

This result, together with the class of functions given in the first part of this Chapter emphasise that certain adding machine transformations have features in common with rotations by irrational numbers with bounded partial quotients. We also note that, as in the $k=1$ case for the irrational rotation, the functions given in the first part are close, in some sense, to those given in the second; however, the discontinuities which give a non-zero integral for the derivative, make all the difference for the cohomology of the cocycle. 


\section{BACKGROUND}

\subsection{Cocycles, Essential Values}

Firstly some definitions and notation in common use throughout this thesis. All of the proofs of the results given here are to be found in [KS].

1.1.1 Definition: Let $(\mathbf{T}, \Omega, \mu)$ denote the one-dimensional torus with standard Borel field and Lebesgue measure. If $T$ is an ergodic automorphism of $\mathbf{T}$, then this defines a $\mathbf{Z}$-action $n \mapsto T^{n}, n \in \mathbf{Z}$ on $(\mathbf{T}, \Omega, \mu)$. A real-valued additive cocycle for $T$ is a Borel map $a: \mathbf{Z} \times \mathbf{T} \rightarrow \mathbf{R}$ satisfying the cocycle relation:

$$
a(n+m, x)=a\left(n, T^{m} x\right)+a(m, x)
$$

for $\mu$-a.e. $x \in \mathbf{T}$, and all $n, m \in \mathbf{Z}$.

Any such cocycle is uniquely determined by the function $f(\cdot)=a(1, \cdot)$. Thus we have

$$
a(n, x)=\left\{\begin{array}{cl}
\sum_{k=0}^{n-1} f \circ T^{k}(\cdot) & \text { for } n \geq 1 \\
0 & \text { for } n=0 \\
-a(-n, \cdot) & \text { for } n \leq-1
\end{array}\right.
$$

Conversely, if $f: \mathbf{T} \rightarrow \mathbf{R}$ is any Borel map, the above formulae will define a real-valued cocycle for $T$. We shall write $f_{n}(x)$ for $a(n, x)$, as defined above, to indicate the relationship between the cocycle and its determining function.

1.1.2 Definition: Let $T$ be an ergodic automorphism of $(\mathbf{T}, \Omega, \mu)$, and $a: \mathbf{Z} \times \mathbf{T} \rightarrow \mathbf{R}$ an additive cocycle for $T$, whose determining function is $f$. The cocycle a will be called a coboundary if and only if there is a Borel map $g: \mathbf{T} \rightarrow \mathbf{R}$ such that

$$
a(1, x)=f(x)=g(T x)-g(x)
$$

for $\mu$-a.e. $x \in \mathbf{T}$. 
1.1.3 Definition: Let $T$ be an ergodic automorphism of $(\mathbf{T}, \Omega, \mu)$ and $a: \mathbf{Z} \times \mathbf{T} \rightarrow \mathbf{R}$ a cocycle for $T$. Let $\overline{\mathbf{R}}=\mathbf{R} \cup\{\infty\}$ be the one-point compactification of $\mathbf{R}$. An element $y \in \overline{\mathbf{R}}$ is called an essential value of a if, for every neighbourhood $N(y)$ of $y$ in $\overline{\mathbf{R}}$ and for every $B \in \Omega$ with $\mu(B)>0$ we have an $n \in \mathbf{Z}$ such that

$$
\mu\left(B \bigcap T^{-n} B \bigcap\{x: a(n, x) \in N(y)\}\right)>0 .
$$

The set of all essential values of the cocycle $a$ is denoted by $\bar{E}(a)$ and we put

$E(a)=\bar{E}(a) \cap \mathbf{R}$.

The following are standard results from $[\mathrm{KS}]$ :

\subsubsection{Lemma: $E(a)$ is a closed (additive) subgroup of $\mathbf{R}$.}

1.1.5 Lemma: The cocycle $a$ is a coboundary if and only if $\bar{E}(a)=\{0\}$.

1.1.6 Lemma: Let $T$ be an ergodic automorphism of $(\mathbf{T}, \Omega, \mu)$ and let $a: \mathbf{Z} \times \mathbf{T} \rightarrow \mathbf{R}$ be a cocycle for $T$. Suppose $\mathcal{K} \subset \mathbf{R}$ is a compact set with $\mathcal{K} \cap E(a)=\emptyset$, then for every $C \in \Omega$ with $\mu(C)>0$ there is a Borel set $B \subset C$ with $\mu(B)>0$ and with

$$
B \bigcap T^{-n} B \bigcap\{x: a(n, x) \in \mathcal{K}\}=\emptyset
$$

for all $n \in \mathbf{Z}$.

\subsection{Diophantine Approximation}

When there is no ambiguity we shall think of arbitrary real numbers as elements of $\mathbf{T}$, by identifying them with their congruence class mod 1 . We shall also employ the following notation: 
1.2.1 Definitions: For $x \in \mathbf{R}$ we denote by $\|x\|$, the distance of $x$ to the nearest integer:

$$
\|x\|=\min \{|j-x|: j \in \mathbf{Z}\} .
$$

For $\alpha \in \mathbf{T}$, we denote by $\left[a_{1}, a_{2}, \ldots\right]$ the continued fraction expansion of $\alpha$. The $a_{k}$ are called the partial quotients of $\alpha$. We define

$$
\frac{p_{k}}{q_{k}}=\left[a_{1}, \ldots, a_{k}\right]=\frac{1}{a_{1}+\frac{1}{a_{2}+\frac{1}{\ldots+\frac{1}{a_{k}}}}}
$$

in lowest terms, for $k \geq 1$. These fractions are called the convergents of $\alpha$, each one being successively a better aproximation (by rational numbers) to $\alpha$, i.e.

$$
\left\|q_{k} \alpha\right\|=\min \left\{|i-j \alpha|: i \in \mathbf{N} \text { and } 0<j<q_{k+1}\right\} ;
$$

for all $k \geq 1$. The $q_{k}$ are called the partial quotient denominators.

These partial quotient denominators tell us which multiples of $\alpha$ are closest to integers. The speed of approximation is related to the growth rate of these denominators. The following lemma makes clear the relationships between the partial quotients and the convergents:

\subsubsection{Lemma:}

(i) $\frac{1}{2}<q_{n}\left\|q_{n-1} \alpha\right\|<1$, for all $n \geq 2$.

(ii) $q_{n}\left\|q_{n} \alpha\right\|<\frac{1}{a_{n+1}}$, for all $n \geq 2$.

(iii) $q_{n+1}=a_{n+1} q_{n}+q_{n-1}$ for all $n \geq 1$ where $q_{0}=1$ and $q_{1}=a_{1}$.

\section{Proof:}

See $[\mathrm{HW}]$ or $[\mathrm{Ca}]$. 


\subsection{The Adding Machine}

As in [H1], we shall consider the following generalisation of the von NeumannKakutani adding machine transformation on $\mathbf{T}$. Let $\mathbf{q}=\left(q_{i}\right)_{i \geq 1}$ be a sequence of integers $q_{i}$, with $q_{i} \geq 2$, for all $i$.

If $\mathbf{A}(\mathbf{q})$ denotes the compact Abelian group of $\mathbf{q}$-adic integers, then the transformation $\mathbf{z} \mapsto \mathbf{z}+\mathbf{1}$ on $\mathbf{A}(\mathbf{q})$, where $\mathbf{1}=(1,0, \ldots)$, is uniquely ergodic with respect to normalised Haar measure on $\mathbf{A}(\mathbf{q})$ (see $[\mathrm{HR}]$ ).

Consider next the circle, $\mathbf{T}$ with Haar measure $\mu$. Define the sequence of integers $p(k), k \geq 0$ by

$$
p(k)=\left\{\begin{array}{cl}
1 & \text { for } k=0 \\
q_{1} \ldots q_{k} & \text { for } k=1,2, \ldots .
\end{array}\right.
$$

If

$$
\mathbf{z}=\sum_{i=0}^{\infty} z_{i} p(i) \text { with } z_{i} \in\left\{0,1, \ldots, q_{i+1}-1\right\}
$$

is an element of $\mathbf{A}(\mathbf{q})$, then

$$
\Phi(\mathbf{z})=\sum_{i=0}^{\infty} \frac{z_{i}}{p(i+1)} \bmod 1
$$

belongs to $\mathbf{T}$. The map $\Phi: \mathbf{A}(\mathbf{q}) \rightarrow \mathbf{T}$ is measure-preserving and injective on $\mathbf{A}(\mathbf{q})$ except on a subset of Haar measure zero.

The $\mathbf{q}$-adic representation of an element $x$ of $\mathbf{T}$,

$$
x=\sum_{i=0}^{\infty} \frac{x_{i}}{p(i+1)} \text { with } x_{i} \in\left\{0,1, \ldots, q_{i+1}-1\right\}
$$

is unique under the condition $x_{i} \neq q_{i+1}-1$ for infinitely many $i$. The uniqueness condition for the representation ensures that the following transformation $T: \mathbf{T} \rightarrow \mathbf{T}$ is well defined:

$$
T x:=\Phi(\mathbf{z}+\mathbf{1}) \text { where } \mathbf{z}=\mathbf{z}(x)=\sum_{i=0}^{\infty} x_{i} p(i) .
$$

$T$ is ergodic with respect to $\mu$ and $T \circ \Phi(\mathbf{z})=\Phi(\mathbf{z}+\mathbf{1})$ for almost all $\mathbf{z} \in \mathbf{A}(\mathbf{q})$. $T$ may be called a (generalised) von Neumann-Kakutani adding machine transformation, with associated integers $p(k), k \geq 1$. 


\subsection{Skew Products}

1.4.1 Definition: Let $(\mathbf{R}, \mathcal{B}, \lambda)$ denote the standard Lebesgue structure on $\mathbf{R}$. We form a new measure space $(\mathbf{T} \times \mathbf{R}, \Omega \times \mathcal{B}, \mu \times \lambda)$ with product structure, suppose that $a: \mathbf{Z} \times \mathbf{T} \rightarrow \mathbf{R}$ is an additive cocycle for the ergodic automorphism $T$ on $\mathbf{T}$, and define a new action $T_{a}$ of $\mathbf{Z}$ on $\mathbf{T} \times \mathbf{R}$ by

$$
T_{a}^{n}(\alpha, x)=\left(T^{n} \alpha, x+a(n, \alpha)\right) .
$$

The $\mathbf{Z}$-action $T_{a}$ on $\mathbf{T} \times \mathbf{R}$ is called the skew product of $T$ with $\mathbf{R}$.

The main result we need comes from $[\mathrm{KS}]$, and relates the ergodicity of $T_{a}$ to the properties of the cocycle:

1.4.2 Theorem: $T_{a}$ is ergodic if and only if $E(a)=\mathbf{R}$.

\subsection{Assorted Results}

We shall make frequent use of the Denjoy-Koksma Inequality:

1.5.1 Theorem: Let $f$ be a real-valued function of bounded variation on $\mathbf{T}$, then there is a positive constant $c=\operatorname{var} f$ such that:

(i) If $T$ denotes the irrational rotation by $\alpha$, which has continued fraction partial quotient denominators $q_{n}$, for $n \geq 1$, then

$$
\sup _{n, x}\left|\sum_{i=0}^{q_{n}-1} f \circ T^{i}(x)-q_{n} \int f d \mu\right| \leq c .
$$

(ii) If $T$ denotes the adding machine transformation with associated integers $p(n)$, for $n \geq 1$, then

$$
\sup _{n, x}\left|\sum_{i=0}^{p(n)-1} f \circ T^{i}(x)-p(n) \int f d \mu\right| \leq c .
$$




\section{Proof:}

For the proof in the case of the irrational rotation, see $[D]$. We give a proof for the adding machine, adapted from the proof given in [He].

For any $n$, let $y_{k}=\frac{k}{p(n)}$ for $k=0, \ldots, p(n)-1$, then the intervals $\left[y_{k}, y_{k+1}\right)$ are $q$-adic cylinders and partition $\mathbf{T}$ into $p(n)$ equal intervals $(n$ cylinders). Consider the points $x_{i}=T^{i} x$ for $i=0, \ldots, p(n)-1$, for some $x \in \mathbf{T}$. Let $\left[y_{k_{i}}, y_{k_{i}+1}\right)$ be the unique interval to which $x_{i}$ belongs, then we have

$$
\left|\sum_{i=0}^{p(n)-1} f \circ T^{i}(x)-p(n) \int f d \mu\right|=\mid \sum_{i=0}^{p(n)-1}\left(f \circ T^{i}(x)-p(n) \int_{y_{k_{i}}}^{y_{k_{i}+1}} f d \mu\right) .
$$

Rearranging the right-hand side and using the triangle inequality yields

$$
\left|\sum_{i=0}^{p(n)-1} p(n) \int_{y_{k_{i}}}^{y_{k_{i}+1}} f\left(T^{i} x\right)-f(t) d \mu(t)\right| \leq \sum_{i=0}^{p(n)-1} p(n) \int_{y_{k_{i}}}^{y_{k_{i}+1}}\left|f\left(T^{i} x\right)-f(t)\right| d \mu(t) .
$$

Hence for all $n$ and all $x \in \mathbf{T}$ we have that

$$
\left|\sum_{i=0}^{p(n)-1} f \circ T^{i}(x)-p(n) \int f d \mu\right| \leq \sum_{i=0}^{p(n)-1} \sup _{t \in\left[y_{k_{i}}, y_{k_{i}+1}\right]}\left|f\left(T^{i} x\right)-f(t)\right| .
$$

This concludes the proof since the right-hand side is bounded above by $c=\operatorname{var} f$.

The following result gives us a condition on the cocycle $f_{n}$, where $f \in L^{p}$, which ensures that it is a coboundary, and its cobounding function belongs to the same $L^{p}$ space.

1.5.2 Theorem: Let $T$ be an ergodic automorphism of $(\mathbf{T}, \Omega, \mu)$ and $f \in L^{p}(\mathbf{T}, \Omega, \mu)$ for some $p$ with $1 \leq p \leq \infty$, and suppose that

$$
c_{0}=\sup _{n}\left\|f_{n}\right\|_{p}<\infty .
$$

Then there exists a function $g \in L^{p}(\mathbf{T}, \Omega, \mu)$ for which $f=g \circ T-g$, and $\|g\|_{p} \leq c_{0}$. 


\section{Proof:}

See $[\mathrm{AS}]$

Finally, we will need to use Weyl's Theorem for both the irrational rotation and the adding machine.

1.5.3 Theorem: Let $f$ be Riemann integrable, and let $T$ denote either the irrational rotation or the adding machine transformation, then

$$
\frac{1}{n} \sum_{i=0}^{n-1} f \circ T^{i}(x) \rightarrow \int f d \mu
$$

uniformly for $\mu$-a.e. $x \in \mathbf{T}$.

\section{Proof:}

For the irrational rotation the result is true for all $x \in \mathbf{T}$, the proof may be found in $[\mathrm{Pe} 2]$. We shall give the proof for the adding machine transformation, which is adapted from the one given in [Pe2].

Since $f$ is Riemann integrable, we have two families of step functions based on q-adic cylinders, $\left\{g_{n}\right\}_{n \geq 1}$ and $\left\{h_{n}\right\}_{n \geq 1}$ with the following properties:

(i) $g_{1}(x) \geq g_{2}(x) \geq \ldots$ and $g_{n} \rightarrow f \mu$-a.e.

(ii) $h_{1}(x) \leq h_{2}(x) \leq \ldots$ and $h_{n} \rightarrow f \mu$-a.e.

We know from [H1, Theorem 2.3] that, for indicator functions $\chi_{I}$ on qadic cylinders $I$, the ergodic average

$$
\frac{1}{n} \sum_{i=0}^{n-1} \chi_{I} \circ T^{i}(x)
$$

converges uniformly to $\mu(I)$ for $\mu$-a.e. $x \in \mathbf{T}$. Hence for all $n$, the step functions $g_{n}, h_{n}$ converge uniformly to their integrals. Therefore, using [Pe2, Remark 4.4(2)] we have that the convergence is also uniform for $f$. 


\section{THE IRRATIONAL ROTATION}

\section{$2.1 \quad$ Introduction}

In this Chapter we study skew product extensions of irrational rotations on $\mathbf{T}$, where the extension is defined by a certain family of classes of realvalued functions. For each $k \geq 1$ we define a class of functions which have $k$ derivatives a.e., satisfying various conditions. We study this class of functions and give, for each $k$, a class of irrational numbers, $\mathcal{S}_{k}$ and show that skew products over rotations by numbers in this class are ergodic.

In section 2, firstly we discuss the orbits of the irrational rotation and give definitions of the classes of functions and irrational numbers that we shall be working with. Then we give a sequence of lemmas about the cocycles defined by functions in our class over rotations by these irrational numbers. Finally we demonstrate that the only possibility for their group of essential values (or asymptotic range) is $\mathbf{R}$, which guarantees ergodicity of the skew product (see 1.4.2).

\subsection{Conditions for ergodicity}

2.2.1 Convention: Throughout this section we shall suppose that $f$ is $k$-times differentiable a.e. $k \geq 1$, with derivatives satisfying the following properties:

(i) $\int f d \mu=0$ and $f^{(k-2)}$ is continuous (if $k>1$ ),

(ii) $f^{(k-1)}$ is piecewise continuous with zero integral,

(iii) $f^{(k)}$ is Riemann integrable with $\int f^{(k)} d \mu \neq 0$.

For the rest of this Chapter we assume without loss of generality that $\int f^{(k)} d \mu>0$, and that the irrational number $\alpha<\frac{1}{2}$.

For small sub-intervals of $\mathbf{T}$ we shall consider the order inherited from $\mathbf{R}$, and use the words left and right accordingly. 
The following result may be found in part 1 , section 4 of $[\mathrm{K}]$.

2.2.2 Proposition: Let $P_{n}(\alpha)$ be the set of right half-open intervals of $\mathbf{T}$ defined by the points $\{-j \alpha\}$ for $j=0, \ldots, q_{n}-1$. Then for all $n$, each interval of $P_{n}(\alpha)$ has length $\left\|q_{n-1} \alpha\right\|$ or $\left\|q_{n} \alpha\right\|+\left\|q_{n-1} \alpha\right\|$. For all $n$, the map $T$ sends each interval of $P_{n}(\alpha)$ onto another, with the following exceptions: for $n$ even

(i) the interval $\left[0,-q_{n-1} \alpha\right)$ is placed inside $\left[-\left(q_{n}-1\right) \alpha,-\left(q_{n-1}-1\right) \alpha\right)$,

(ii) the interval $\left[-\left(q_{n}-q_{n-1}\right) \alpha, 0\right)$ overflows $\left[-\left(q_{n}-q_{n-1}-1\right) \alpha,-\left(q_{n}-1\right) \alpha\right)$.

For odd $n$ the intervals are the same but the end-points are in a different order.

For $j=0, \ldots, k-1$ we denote the variation of $f^{(j)}$ by $c(j)$, then the Denjoy-Koksma inequality 1.5.1 gives us that

$$
\left|f_{q_{n}}^{(j)}(x)\right|<c(j),
$$

for each $j=0, \ldots, k-1$, all $n \geq 0$ and all $x \in \mathbf{T}$.

Let $\omega_{1}, \ldots, \omega_{N}$ be the discontinuities of $f^{(k-1)}$. Arrange these discontinuities in increasing order: $0 \leq \omega_{1} \leq \ldots \leq \omega_{N}<1$. We may immediately assume that $\omega_{1}=0$, since the rotation of the domain of $f$ necessary to bring this about may be performed initially, and does not affect the ergodicity in question.

Suppose now that at $\omega_{r}, f^{(k-1)}$ jumps by $d_{r}=f^{(k-1)}\left(\omega_{r}^{+}\right)-f^{(k-1)}\left(\omega_{r}^{-}\right)$ for $r=1, \ldots, N$, where $f^{(k-1)}\left(\omega_{r}^{+}\right), f^{(k-1)}\left(\omega_{r}^{-}\right)$are the limits of $f^{(k-1)}(x)$ as $x$ approaches $\omega_{r}$ from the left, right respectively. Since $f^{(k-1)}$ is piecewise continuous we have the following relationship between $\int f^{(k)} d \mu$ and these jumps:

\subsubsection{Lemma:}

$$
\int f^{(k)} d \mu=\sum_{r=1}^{N} d_{r}
$$


Hence the condition for $\int f^{(k)} d \mu \neq 0$ is equivalent to $\sum d_{r} \neq 0$. We also have the following:

2.2.4 Proposition: For all $n$, the graph of the cocycle $f_{q_{n}}^{(k-1)}$ has discontinuities at $\left\{w_{r}-j \alpha\right\}$ for $0 \leq j<q_{n}$ and $r=1, \ldots, N$, with jumps of size $d_{r}$ at these points. Also, each partition interval $Q_{i}^{n}$ of $P_{n}(\alpha), i=1, \ldots, q_{n}$, contains at most $2 N-2$ discontinuities of $f_{q_{n}}^{(k-1)}$ for all $n$.

\section{Proof:}

The first part is clear. For the second part we show that for all $n$ and each discontinuity $w_{r}$ for $r=2, \ldots, N$ of $f^{(k-1)}$, there can be at most two discontinuities of $f_{q_{n}}^{(k-1)}$ due to it in any partition interval $Q_{i}^{n}$ of $P_{n}(\alpha)$.

By the first part, for fixed $r$ and all $n$, the discontinuities of $f_{q_{n}}^{(k-1)}$ due to $\omega_{r}$ occur at the points $\left\{\omega_{r}-j \alpha\right\}$ for $0 \leq j<q_{n}$. Their spacing is the same as for $P_{n}(\alpha)$ (since this is the set $P_{n}(\alpha)$ shifted by $\left.\omega_{r}\right)$. The distance between two discontinuities due to $\omega_{r}$ is thus $\left\|q_{n-1} \alpha\right\|$ or $\left\|q_{n-1} \alpha\right\|+\left\|q_{n} \alpha\right\|$ from Proposition 2.2.2. These distances are also the two possible sizes of $Q_{i}^{n}$. Now from 1.2.1 we have for all $n$, that

$$
\left\|q_{n-1} \alpha\right\|<\left\|q_{n-1} \alpha\right\|+\left\|q_{n} \alpha\right\|<2 .\left\|q_{n-1} \alpha\right\| .
$$

We see that if $Q_{i}^{n}$ has length $\left\|q_{n-1} \alpha\right\|+\left\|q_{n} \alpha\right\|$, then at most two discontinuities due to $\omega_{r}$ of separation $\left\|q_{n-1} \alpha\right\|$ may be placed inside the interval. If $Q_{i}^{n}$ has length $\left\|q_{n-1} \alpha\right\|$ then, clearly at most one discontinuity due to $\omega_{r}$ may fall inside it. Thus the maximum number of discontinuities of $f_{q_{n}}^{(k-1)}$ which may occur in any interval of $P_{n}(\alpha)$ is $2(N-1)$. This implies the assertion.

2.2.5 Definition: For any positive integer $k \geq 1$, define the subset $\mathcal{S}_{k}$ of irrational numbers whose partial quotients $\left\{a_{n}\right\}, n \geq 1$ satisfy the following condition:

$$
\limsup \frac{a_{n+1}}{q_{n}^{k-1}}>0
$$

For any $\alpha \in \mathcal{S}_{k}$, suppose that $\lim \sup \frac{a_{n+1}}{q_{n}^{k-1}}=S>0$, so there exists a subsequence of integers $\left\{n_{j}\right\}_{j \geq 1}$ and an integer $J$, with $a_{n_{j}+1} \geq K q_{n_{j}}^{k-1}$ for some $0<K<S$ and all $j>J$. Since this is the subsequence we shall be 
considering, for convenience we drop the subscripts and assume that there is a positive constant $K$ and an integer $N^{\prime}$ such that

$$
a_{n+1} \geq K q_{n}^{k-1} \text { for } n \geq N^{\prime} .
$$

For the rest of this Chapter we shall assume that $\alpha \in \mathcal{S}_{k}$.

2.2.6 Lemma: There is a positive constant $Y$, such that for $n>N^{\prime}$ and $j=0, \ldots, k-1$

$$
\left|f_{q_{n}}^{(j)}(x)\right|<\frac{Y}{q_{n}^{k-1-j}}
$$

for all $x \in \mathbf{T}$.

\section{Proof:}

The result is certainly true for $j=k-1$ from (2) above, for all $n$, with $Y=Y(k-1)=c(k-1)$. We shall suppose that the formula holds for some $1 \leq j \leq k-1$ with $Y=Y(j)$ and demonstrate that this implies the inequality for $j-1, n>N^{\prime}$ and some $Y=Y(j-1)$. Then, if we take $Y$ to be the maximum of all these $Y(j)$ for $j=0, \ldots, k-1$ this will prove our result.

We proceed in three steps: firstly we show that there is a positive constant $E$ such that for $n>N^{\prime}$, and all intervals $Q_{i}^{n} \in P_{n}(\alpha)$

$$
\left|\int_{Q_{i}^{n}} f_{q_{n}}^{(j-1)} d \mu\right|<\frac{E}{q_{n}^{k}} .
$$

From Proposition 2.2.2 and the cocycle relation (1), the integral in question is the integral of $f^{(j-1)}$ over $q_{n}-1$ iterates of $Q_{i}^{n}$ under $T$. Suppose that $\mu\left(Q_{i}^{n}\right)=\left\|q_{n-1} \alpha\right\|$, then the integral is equal to the integral of $f^{(j-1)}$ over all of $\mathbf{T}$, except for $q_{n-1}$ intervals of size $\left\|q_{n} \alpha\right\|$ which are iterates under $T$. Hence

$$
\int_{Q_{i}^{n}} f_{q_{n}}^{(j-1)} d \mu=\int_{\mathbf{T}} f^{(j-1)} d \mu-\int_{I} f_{q_{n-1}}^{(j-1)} d \mu
$$

where $I$ is the interval of size $\left\|q_{n} \alpha\right\|$, whose $q_{n-1}$ iterates are missed out. Thus we have 


$$
\left|\int_{Q_{i}^{n}} f_{q_{n}}^{(j-1)} d \mu\right|=\left|\int_{I} f_{q_{n-1}}^{(j-1)} d \mu\right|
$$

since $f^{(j-1)}$ has zero integral over T. Applying (2) and 1.2.2ii), we get

$$
\left|\int_{Q_{i}^{n}} f_{q_{n}}^{(j-1)} d \mu\right| \leq c(j-1)\left\|q_{n} \alpha\right\|<\frac{c(j-1)}{q_{n} a_{n+1}}<\frac{c(j-1)}{K q_{n}^{k}},
$$

for $n>N^{\prime}$, since $\alpha \in \mathcal{S}_{k}$.

If $\mu\left(Q_{i}^{n}\right)=\left\|q_{n-1} \alpha\right\|+\left\|q_{n} \alpha\right\|$, then we split this interval into two chunks, one $I_{1}$ of size $\left\|q_{n-1} \alpha\right\|$, the other $I_{2}$ of size $\left\|q_{n} \alpha\right\|$. For $I_{1}$ we get the same estimate as above for the integral of $f_{q_{n}}^{(j-1)}$ over it. For $I_{2}$ we have that for $n>N^{\prime}$

$$
\left|\int_{I_{2}} f_{q_{n}}^{(j-1)} d \mu\right|<c(j-1)\left\|q_{n} \alpha\right\|<\frac{c(j-1)}{K q_{n}^{k}}
$$

also by (2) and 1.2.2ii). Therefore, if we choose $E=\frac{2 c(j-1)}{K}$, then we have established (5) for all intervals $Q_{i}^{n} \in P_{n}(\alpha)$, and all $n>N^{\prime}$.

For the second step we demonstrate the following: there is a positive constant $G$ such that for $n>N^{\prime}$, in each interval $Q_{i}^{n} \in P_{n}(\alpha)$ there is an $x \in Q_{i}^{n}$ with

$$
\left|f_{q_{n}}^{(j-1)}(x)\right|<\frac{G}{q_{n}^{k-1}} .
$$

Suppose not, then for some $Q_{i}^{n} \in P_{n}(\alpha)$ and any $G>0$ we have that $\left|f_{q_{n}}^{(j-1)}(x)\right| \geq \frac{G}{q_{n}^{k-1}}$ for all $x \in Q_{i}^{n}$ and some $n>N^{\prime}$. In particular this holds for $G=4 E$. Supposing, without loss of generality that $f_{q_{n}}^{(j-1)}$ is strictly positive on $Q_{i}^{n}$, we must have that

$$
\left|\int_{Q_{i}^{n}} f_{q_{n}}^{(j-1)} d \mu\right|>\frac{G}{q_{n}^{k-1}} \cdot \mu\left(Q_{i}^{n}\right) \geq \frac{G}{q_{n}^{k-1}} \cdot\left\|q_{n-1} \alpha\right\|,
$$

from Proposition 2.2.2. However, from (5) we have an upper bound for this integral, and since $n>N^{\prime}$, we get 


$$
\frac{G}{q_{n}^{k-1}} \cdot\left\|q_{n-1} \alpha\right\|<\frac{E}{q_{n}^{k}}
$$

Hence we have that

$$
q_{n} \cdot\left\|q_{n-1} \alpha\right\|<\frac{E}{G}=\frac{1}{4}
$$

which contradicts 1.2.2i). This establishes (6).

Finally we show the following: there is a constant $Y(j-1)$ such that for $n>N^{\prime}$, and all intervals $Q_{i}^{n}$ of $P_{n}(\alpha)$

$$
\left|f_{q_{n}}^{(j-1)}(x)\right|<\frac{Y(j-1)}{q_{n}^{k-j}}
$$

for all $x \in Q_{i}^{n}$.

Since $f^{(j-1)}$ is absolutely continuous and the formula is true for $j$ we have, for all $a, b \in \mathbf{T}$, that

$$
\left|f_{q_{n}}^{(j-1)}(b)-f_{q_{n}}^{(j-1)}(a)\right|=\left|\int_{a}^{b} f_{q_{n}}^{(j)} d \mu\right|<\frac{Y(j)}{q_{n}^{k-1-j}} \cdot|b-a| .
$$

By (3) and 1.2.2i), for $a, b \in Q_{i}^{n}$ this becomes

$$
\left|f_{q_{n}}^{(j-1)}(b)-f_{q_{n}}^{(j-1)}(a)\right|<\frac{Y(j)}{q_{n}^{k-1-j}} \cdot \mu\left(Q_{i}^{n}\right)<\frac{2 Y(j)}{q_{n}^{k-j}} .
$$

From (6) we may choose $a$ to be a point for which $\left|f_{q_{n}}^{(j-1)}(a)\right|<\frac{G}{q_{n}^{k-1}}$, hence we have for $n>N^{\prime}$ and all $x \in Q_{i}^{n}$, that

$$
\left|f_{q_{n}}^{(j-1)}(x)\right|<\frac{G}{q_{n}^{k-1}}+\frac{2 Y(j)}{q_{n}^{k-j}} \leq \frac{2 Y(j)+G}{q_{n}^{k-1}} .
$$

This proves the result for $j-1, n>N^{\prime}$ with $Y(j-1)=2 Y(j)+G$, and the rest follows by induction. 
2.2.7 Lemma: There are positive constants $K_{1}(k), K_{2}(k)$ and a positive integer $Z$ such that for $n>Z$ we have

$$
n . K_{1}(k)<f_{n}^{(k)}(x)<n . K_{2}(k)
$$

for all $x \in \mathbf{T}$.

\section{Proof:}

From the definition of $f^{(k)}$ given in 2.2.1, and using Weyl's theorem 1.5.3, we have that

$$
\frac{1}{n} \sum_{i=0}^{n-1} f^{(k)} \circ T^{i}(x) \rightarrow \int f^{(k)} d \mu
$$

uniformly, for all $x \in \mathbf{T}$. Given this, there is a positive integer $Z$, such that for $n>Z$ we obtain

$$
\left|\frac{f_{n}^{(k)}(x)}{n}-\int f^{(k)} d \mu\right|<\frac{1}{2} \int f^{(k)} d \mu
$$

for all $x \in \mathbf{T}$. So

$$
K_{1}(k)<\frac{f_{n}^{(k)}(x)}{n}<K_{2}(k)
$$

for all $x \in \mathbf{T}$, where $K_{1}(k)=\frac{1}{2} \int f^{(k)} d \mu$ and $K_{2}(k)=\frac{3}{2} \int f^{(k)} d \mu$.

2.2.8 Proposition: Suppose that $g$ is a function which defines the cocycle $g_{q_{n}}$, for which there are positive constants $R, L_{1}, L_{2}$ and an integer $N_{1}$ such that for $n>N_{1}$, all partition intervals $Q_{i}^{n}$ of $P_{n}(\alpha)$ contain a subinterval $J_{i}^{n}=\left[a_{i}^{n}, b_{i}^{n}\right)$ which satisfies $\mu\left(J_{i}^{n}\right) \geq \frac{1}{R} \mu\left(Q_{i}^{n}\right)$ on which $g_{q_{n}}$ is absolutely continuous and

$$
q_{n} \cdot L_{1}<\left|g_{q_{n}}^{\prime}(x)\right|<q_{n} \cdot L_{2}
$$

for $n>N_{1}$ and all $x \in J_{i}^{n}$. Then there are constants $H, F$ independent of $i$ and $n$, such that for $n>N_{1}$,

(i) $g_{q_{n}}$ moves through a height greater than $H$ on $J_{i}^{n}$,

(ii) for any interval $I_{i}^{n}=[x, y] \subseteq g_{q_{n}}\left(J_{i}^{n}\right)$ we have that

$$
\frac{\mu\left(g_{q_{n}}^{-1}\left(I_{i}^{n}\right) \cap J_{i}^{n}\right)}{\mu\left(Q_{i}^{n}\right)}>F|y-x| .
$$




\section{Proof:}

Choose $H=\frac{L_{1}}{4 R}$, and suppose that $g_{q_{n}}$ is strictly increasing for $n>N_{1}$, then the height, $h$ moved through by the cocycle on $J_{i}{ }^{n}$ is given by

$$
h=g_{q_{n}}\left(b_{i}^{n}\right)-g_{q_{n}}\left(a_{i}^{n}\right)=\int_{a_{i}^{n}}^{b_{i}^{n}} g_{q_{n}}^{\prime} d \mu .
$$

By hypothesis, and applying Proposition 2.2.2, we have that for $n>N_{1}$

$$
h>\frac{L_{1}}{R} \cdot q_{n} \cdot \mu\left(Q_{i}^{n}\right)>\frac{L_{1}}{2 R} .
$$

If $g_{q_{n}}$ is strictly decreasing we obtain, also for $n>N_{1}$, a reversed inequality, but with a minus sign, this proves i).

For ii), define $m=g_{q_{n}}^{-1}(x) \cap J_{i}^{n}$ and $p=g_{q_{n}}^{-1}(y) \cap J_{i}^{n}$ then, supposing that $g_{q_{n}}$ is strictly increasing, for $n>N_{1}$ we have that $g_{q_{n}}^{-1}\left(I_{i}^{n}\right) \cap J_{i}^{n}=[m, p)$, and

$$
y-x=\int_{m}^{p} g_{q_{n}}^{\prime} d \mu .
$$

By definition of $g_{q_{n}}^{\prime}$ we have, for $n>N_{1}$, that

$$
p-m>\frac{y-x}{q_{n} \cdot L_{2}}
$$

Hence from (3) we obtain

$$
\frac{p-m}{\mu\left(Q_{i}^{n}\right)}>\frac{y-x}{2 L_{2} \cdot q_{n}\left\|q_{n-1} \alpha\right\|},
$$

and so

$$
\frac{\mu\left(g_{q_{n}}^{-1}\left(I_{i}^{n}\right) \cap J_{i}^{n}\right)}{\mu\left(Q_{i}^{n}\right)}>\frac{y-x}{2 L_{2}} .
$$

If $g_{q_{n}}$ is strictly decreasing, we get the same inequality for $n>N_{1}$, so putting $F=\frac{1}{2 L_{2}}$ completes the proof of ii). 
2.2.9 Lemma: For $j=0, \ldots, k-1$ there exist strictly positive constants $H(j), F(j), L(j)$ and an integer $N_{1}(j)$ with the following properties: within each interval $Q_{i}^{n}$ of $P_{n}(\alpha)$ there is a subinterval $J_{i}^{n}(j)$, with $\mu\left(J_{i}^{n}(j)\right)>\frac{1}{L(j)} \mu\left(Q_{i}^{n}\right)$ such that for $n>N_{1}(j)$,

(i) $f_{q_{n}^{k-j}}^{(j)}$ moves through a height greater than $H(j)$ on $J_{i}^{n}(j)$,

(ii) for any interval $I_{i}^{n}(j)=[x, y]$ in $f_{q_{n}^{k-j}}^{(j)}\left(J_{i}^{n}(j)\right)$ we have that

$$
\frac{\mu\left(\left(f_{q_{n}^{k-j}}^{(j)}\right)^{-1}\left(I_{i}^{n}(j)\right) \cap J_{i}^{n}(j)\right)}{\mu\left(Q_{i}^{n}\right)}>F(j)|y-x| .
$$

\section{Proof:}

The result is certainly true for $j=k-1$ by using Lemma 2.2.7, Proposition 2.2.4, and applying Proposition 2.2.8 to $g=f^{(k-1)}$ with $R=\frac{1}{2 N-2}$, $L_{1}=K_{1}(k), L_{2}=K_{2}(k)$ and $N_{1}$ such that $q_{N_{1}}>Z$. We shall assume that the result is true for some $1 \leq j \leq k-1$, and show that this implies that the result holds for $j-1$.

From i) above, since the result is true for $j$, we know that for $n>N_{1}(j)$, $f_{q_{n}^{k-j}}^{(j)}$ moves through a height greater than $H(j)$ on every $J_{i}^{n}(j) \subseteq Q_{i}^{n}$ for $i=1, \ldots, q_{n}$, where $\mu\left(J_{i}^{n}(j)\right) \geq \frac{1}{L(j)} \mu\left(Q_{i}^{n}\right)$. Without loss of generality we may assume that there is some $x \in J_{i}^{n}(j)$ with $f_{q_{n}^{k-j}}^{(j)}(x)>\frac{1}{4} H(j)$. As the result is true for $j$, we may apply ii) above to the interval

$$
I_{i}^{n}(j)=\left[\frac{1}{4} H(j), Y\right] \cap f_{q_{n}^{k-j}}^{(j)}\left(J_{i}^{n}(j)\right) .
$$

Hence for $n>N_{1}(j)$, there is a subinterval $S_{i}^{n}(j)$ in each $J_{i}^{n}(j)$ which satisfies $\mu\left(S_{i}^{n}(j)\right)>\frac{1}{L^{\prime}(j)} \mu\left(Q_{i}^{n}\right)$, and on which

$$
t_{1}(j)<\left|f_{q_{n}^{k-j}}^{(j)}(x)\right|<t_{2}(j)
$$

for some strictly positive constants $t_{1}(j), t_{2}(j)$ and $L^{\prime}(j)$.

From the cocycle relation (1), for all $n$ and all $x \in \mathbf{T}$, we have that 


$$
f_{q_{n}^{k-j+1}}^{(j)}(x)=f_{q_{n}^{k-j}}^{(j)}(x)+f_{q_{n}^{k-j}}^{(j)}\left(T^{q_{n}} x\right)+\ldots+f_{q_{n}^{k-j}}^{(j)}\left(T^{q_{n}\left(q_{n}-1\right)} x\right) .
$$

Hence, in order to estimate bounds for $f_{q_{n}^{k-j+1}}^{(j)}(x)$ we must look at the values of $f_{q_{n}^{k-j}}^{(j)}$ for $q_{n}-1$ iterates of $x$ by $T^{q_{n}}$. Note that, since $\alpha \in \mathcal{S}_{k}$ we have, for $n>N^{\prime}$

$$
\left|T^{q_{n}} x-x\right|=\left\|q_{n} \alpha\right\|<\frac{1}{K q_{n}^{k}}
$$

and so

$$
\left|T^{q_{n}\left(q_{n}-1\right)} x-x\right|<\frac{1}{K q_{n}^{k-1}} .
$$

Hence for $n>N_{1}(j-1)=\max \left\{N^{\prime}, N_{1}(j)\right\}$, all points in $S_{i}^{n}(j)$, except for a subinterval $I$ at one end, of size $\mu(I)<\frac{1}{K q_{n}^{k-1}}$, return to $S_{i}^{n}(j)$ at least $q_{n}-1$ times under $T^{q_{n}}$. Thus from (7), for $x \in V_{i}^{n}(j)=S_{i}^{n}(j) \backslash I$ we have that

$$
q_{n} \cdot t_{1}(j)<\left|f_{q_{n}^{k-j+1}}^{(j)}(x)\right|<q_{n} \cdot t_{2}(j)
$$

for $n>N_{1}(j-1)$.

We may now apply Proposition 2.2.8 for $g=f_{q_{n}^{k-j}}^{(j-1)}$ and the interval $I_{i}^{n}=V_{i}^{n}(j)$ to obtain our result for $j-1$.

By induction the result holds for $j=0, \ldots, k-1$. 
2.2.10 Lemma: For any $A \subseteq \mathbf{T}$ with $\mu(A)>0$, and every $\epsilon>0$, there is an $A_{0} \subseteq A$ with $\mu\left(A \backslash A_{0}\right)<\epsilon$ and an infinite sequence $\left\{n_{i}\right\}_{i \geq 1}$ of integers such that $T^{q_{n_{i}}^{k}} x \in A$ for all $i$, and all $x \in A_{0}$.

\section{Proof:}

For any Borel set $B$ of positive measure the map

$$
x \mapsto \mu(B \bigcap(B-x))
$$

is continuous at 0 , where $B-x$ denotes the set $B$ translated by the element $-x \in \mathbf{T}$ (c.f. [R, Theorem 1.1.5]).

So, given $\epsilon>0$, there is a $\delta>0$ such that $|\mu(B)-\mu(B \cap(B-x))|<\epsilon$ for $|x|<\delta$. Firstly we show that $\left\|q_{n}^{k} \alpha\right\| \rightarrow 0$ as $n \rightarrow \infty$. By definition,

$$
\begin{aligned}
\left\|q_{n}^{k} \alpha\right\| & =\min \left\{\left|j-q_{n}^{k} \alpha\right|: j \in \mathbf{Z}\right\} \\
& =\min \left\{\left|j-(\underbrace{q_{n} \alpha+\ldots+q_{n} \alpha}_{q_{n}^{k-1} \text { times }})\right|: j \in \mathbf{Z}\right\}
\end{aligned}
$$

so, by the triangle inequality,

$$
\begin{gathered}
\leq \min \left\{\left|j_{1}-q_{n} \alpha\right|+\ldots+\left|j_{q_{n}^{k-1}}-q_{n} \alpha\right|: j_{1}, \ldots, j_{q_{n}^{k-1}} \in \mathbf{Z}\right\} \\
=q_{n}^{k-1}\left\|q_{n} \alpha\right\| .
\end{gathered}
$$

From (4) we have that

$$
q_{n}^{k-1} \leq \frac{a_{n+1}}{K}
$$

for $n>N^{\prime}$, so we obtain

$$
\left\|q_{n}^{k} \alpha\right\| \leq \frac{a_{n+1}}{K}\left\|q_{n} \alpha\right\| .
$$

From 1.2.2ii) for all $n$ we have

$$
\left\|q_{n} \alpha\right\|<\frac{1}{q_{n} a_{n+1}},
$$


hence

$$
\left\|q_{n}^{k} \alpha\right\|<\frac{1}{K q_{n}} \rightarrow 0 \quad \text { as } n \rightarrow \infty .
$$

So given $\delta>0$ we may choose $K^{\prime \prime}>0$ such that for $n>K^{\prime \prime}$ we have $\left\|q_{n}^{k} \alpha\right\|<\delta$, and hence

$$
|\mu(B)-\mu(B \bigcap(B-x))|=\left|\mu(B)-\mu\left(B \bigcap T^{-q_{n}^{k}} B\right)\right|<\epsilon .
$$

If we apply the above to our set $\mathrm{A}$, given $\epsilon>0$ we can find an $n_{1}$ such that

$$
\mu\left(A \bigcap T^{-q_{n_{1}}^{k}} A\right)>\mu(A)-\frac{\epsilon}{2} .
$$

Now we apply the above argument to the set $A \cap T^{-q_{n_{1}}^{k}} A$ and obtain an $n_{2}$ with

$$
\mu\left(A \bigcap T^{-q_{n_{1}}^{k}} A \bigcap T^{-q_{n_{2}}^{k}} A \bigcap T^{-q_{n_{1}}^{k}-q_{n_{2}}^{k}} A\right)>\mu\left(A \bigcap T^{-q_{n_{1}}^{k}} A\right)-\frac{\epsilon}{4} .
$$

Then by monotonicity, (8) and (9) we have that

$$
\begin{gathered}
\mu\left(A \bigcap T^{-q_{n_{1}}^{k}} A \bigcap T^{-q_{n_{2}}^{k}} A\right) \geq \mu\left(A \bigcap T^{-q_{n_{1}}^{k}} A \bigcap T^{-q_{n_{2}}^{k}} A \bigcap T^{-q_{n_{1}}^{k}-q_{n_{2}}^{k}} A\right) \\
>\mu\left(A \bigcap T^{-q_{n_{1}}^{k}} A\right)-\frac{\epsilon}{4}>\mu(A)-\frac{3}{4} \epsilon .
\end{gathered}
$$

Inductively, we get for $i \geq 1$

$$
\mu\left(A \bigcap T^{-q_{n_{1}}^{k}} A \bigcap \ldots \bigcap T^{-q_{n_{i}}^{k}} A\right)>\mu(A)-\left(\frac{2^{i}-1}{2^{i}}\right) \epsilon .
$$

Letting $i \rightarrow \infty$ and defining $q_{n_{0}}=0$, we have that

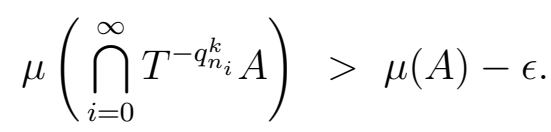

The set $A_{0}=\bigcap_{i=0}^{\infty} T^{-q_{n_{i}}^{k}} A$ has the required properties. 
2.2.11 Lemma: Given $A \subseteq \mathbf{T}$ with $\mu(A)>0$, and $\epsilon^{\prime}>0$ there is an $A_{0} \subseteq A$ with $\mu\left(A_{0}\right)>0$ and $\mu\left(A \backslash A_{0}\right)<\epsilon^{\prime}$, with the following properties: Given any

$\epsilon>0$, there exists $a \delta>0$ such that for all $x \in A_{0}$, and all intervals $I(x)$ containing $x$ of length $\mu(I(x))<\delta$, we have that

$$
\frac{\mu(I(x) \cap A)}{\mu(I(x))}>1-\epsilon .
$$

\section{Proof:}

Define, for $n \geq 1$, a sequence of functions $g_{n}$ on $\mathbf{T}$ by

$$
g_{n}(x)=\inf \frac{\mu\left(I_{n}(x) \cap A\right)}{\mu\left(I_{n}(x)\right)}
$$

where the infimum is taken over all intervals $I_{n}(x) \subset \mathbf{T}$ containing $x$ which satisfy $\mu\left(I_{n}(x)\right)<\frac{1}{n}$. By a similar argument to that given in the proof of Lemma 2.2.10, the function

$$
g(x, \beta, \gamma)=\frac{\mu((x-\beta, x-\gamma) \cap A)}{\gamma+\beta}
$$

is continuous in $x$, for any $\beta, \gamma>0$. Now $g_{n}$ defined above is the infimum of such functions, with the condition that $|\beta+\gamma|<\frac{1}{n}$. From [C, p.229] we see that $g_{n}$ is upper semi-continuous, and therefore Borel measureable.

The Lebesgue Density Theorem says that $g_{n}(x) \rightarrow 1$ for almost all $x \in A$ and $g_{n}(x) \rightarrow 0$ for almost all $x \in A^{c}$. Hence the Borel function $g=\liminf g_{n}$ is almost everywhere equal to the characteristic function of $A$.

By Egoroff's Theorem, given $\epsilon^{\prime}>0$, there is a Borel set $A_{0} \subseteq A$ with $\mu\left(A \backslash A_{0}\right)<\epsilon^{\prime}$ such that $g_{n} \rightarrow g$ uniformly on $A_{0}$. That is, given $\epsilon>0$, there is an $N \in \mathbf{N}$ such that

$$
\left|g_{n}(x)-g(x)\right|<\epsilon
$$

for all $x \in A_{0}$, and all $n \geq N$. Thus we have for such $n$,

$$
g_{n}(x)>1-\epsilon
$$

for all $x \in A_{0}$, since $g$ is the characteristic function of $A$. By definition of $g_{n}$ this implies that for $n \geq N$ 


$$
\inf \frac{\mu\left(I_{n}(x) \cap A\right)}{\mu\left(I_{n}(x)\right)}>1-\epsilon .
$$

So, putting $\delta=\frac{1}{N}$, we have our result.

\subsubsection{Lemma: $E(f) \neq \lambda \mathbf{Z}$ for any $\lambda \geq 0$.}

\section{Proof:}

Suppose that $E(f)=\lambda \mathbf{Z}$ where $\lambda \geq 0$. By the cocycle relation (1) and Lemma 2.2.6 we have that $\left|f_{q_{n}^{k}}(x)\right|<Y$ for all $n>N^{\prime}$, and all $x \in \mathbf{T}$. Let $v$ be the greatest integer such that $v \lambda<Y$. We choose

$$
0<\epsilon<\frac{H(0)}{4(2 v+1)}
$$

where $H(0)$ is the number obtained in Lemma 2.2.9i). Consider the compact set

$$
\mathcal{K}=(-\epsilon, Y] \backslash \bigcup_{i=0}^{v}(i \lambda-\epsilon, i \lambda+\epsilon) .
$$

This is the interval $(-\epsilon, Y]$ without sub-intervals of width $2 \epsilon$ about each $i \lambda \in E(f)$, for $i=0, \ldots, v$. Clearly $\mathcal{K} \cap E(f)=\emptyset$, so applying Proposition 1.1.6 we have that there is a Borel set $B \in \Omega$ with $\mu(B)>0$ such that for all $m \in \mathbf{Z}, B \cap T^{-m} B \cap\left\{y:\left|f_{m}(y)\right| \in \mathcal{K}\right\}=\emptyset$.

From Lemma 2.2.9i), for all $n>N_{1}(0)$, every interval, $Q_{i}^{n}$ in $P_{n}(\alpha)$ contains a subinterval $J_{i}^{n}(0)$ on which $\left|f_{q_{n}^{k}}\right|$ moves continuously through a height greater than $\frac{1}{2} H(0)$. We claim that for $n$ sufficiently large, and for every interval $Q_{i}^{n}$, the ratio of the Lebesgue measure of $Q_{i}^{n}$ to that of the set $Q_{i}^{n} \cap\left\{y:\left|f_{q_{n}^{k}}(y)\right| \in \mathcal{K}\right\}$ is greater than a fixed positive number.

Applying 2.2.9ii) to the interval(s) comprising $\mathcal{K}$ of total length at least $\frac{1}{2} H(0)-(2 v+1) \epsilon>\frac{1}{4} H(0)$, through which the graph of $\left|f_{q_{n}^{k}}\right|$ must pass, gives us a strictly positive constant $F$, independent of $i$, such that, for $n>N_{1}(0)$,

$$
\frac{\mu\left(J_{i}^{n}(0) \cap\left\{y:\left|f_{q_{n}^{k}}(y)\right| \in \mathcal{K}\right\}\right)}{\mu\left(Q_{i}^{n}\right)}>F \frac{H(0)}{4} .
$$


So, by monotonicity, for $n>N_{1}(0)$ there exists a strictly positive constant $W$, independent of $i$, such that

$$
\frac{\mu\left(Q_{i}^{n} \cap\left\{y:\left|f_{q_{n}^{k}}(y)\right| \in \mathcal{K}\right\}\right)}{\mu\left(Q_{i}^{n}\right)}>W .
$$

This proves our claim.

Now from Lemma 2.2.11, given $0<\epsilon^{\prime}<\frac{1}{2} \mu(B)$, there is a $B_{0} \subseteq B$ with $\mu\left(B \backslash B_{0}\right)<\epsilon^{\prime}$, and a $\delta>0$, such that, for all $x \in B_{0}$,

$$
\frac{\mu\left(I^{\prime}(x) \bigcap B\right)}{\mu\left(I^{\prime}(x)\right)}>1-\frac{1}{2} W
$$

for any interval $I^{\prime}(x)$ containing $x$, of length $\mu\left(I^{\prime}(x)\right)<\delta$. Then, from Lemma 2.2.10, given $0<\epsilon^{\prime \prime}<\frac{1}{2} \mu\left(B_{0}\right)$, there is a Borel set $B_{1} \subseteq B_{0}$ with $\mu\left(B_{0} \backslash B_{1}\right)<\epsilon^{\prime \prime}$, and a sequence $\left\{n_{j}\right\}_{j \geq 1}$ of integers, with $T^{q_{n_{j}}^{k}} x \in B_{0}$ for all $x \in B_{1}$. We now fix $x \in B_{1}$. From Proposition 2.2 .2 we know that, given $\delta>0$ there is an integer $M^{\prime}$ such that, for $k>M^{\prime}$,

$$
\mu\left(Q_{i_{k}}^{k}(x)\right)<\delta
$$

where $Q_{i_{k}}^{k}(x)$ is the interval in $P_{k}(\alpha)$ which contains $x$. So for $n=n_{j}>M^{\prime}$, we have that

$$
\frac{\mu\left(Q_{i}^{n}(x) \cap B\right)}{\mu\left(Q_{i}^{n}(x)\right)}>1-\frac{1}{2} W .
$$

Now, applying (11) to $T^{q_{n}^{k}} x \in B_{0}$ for this $n$, we also get that

$$
\frac{\mu\left(T^{q_{n}^{k}} Q_{i}^{n}(x) \cap B\right)}{\mu\left(T^{q_{n}^{k}} Q_{i}^{n}(x)\right)}>1-\frac{1}{2} W
$$

since the interval $T_{n}^{q_{n}^{k}} Q_{i}^{n}(x)$ encloses the point $T_{n}^{q_{n}^{k}} x$ and

$$
\mu\left(T^{q_{n}^{k}} Q_{i}^{n}(x)\right)=\mu\left(Q_{i}^{n}(x)\right)<\delta .
$$

So, using the $T$-invariance of $\mu,(13)$ becomes

$$
\frac{\mu\left(Q_{i}^{n}(x) \cap T^{-q_{n}^{k}} B\right)}{\mu\left(Q_{i}^{n}(x)\right)}>1-\frac{1}{2} W .
$$


By (12) and (14) $B$ and $T^{-q_{n}^{k}} B$ take up, in proportion, more than $1-\frac{1}{2} W$ of $Q_{i}^{n}(x)$ for $n=n_{j}>M^{\prime}$. Hence we have that

$$
\frac{\mu\left(Q_{i}^{n}(x) \cap B \cap T^{-q_{n}^{k}} B\right)}{\mu\left(Q_{i}^{n}(x)\right)}>1-W
$$

for $n=n_{j}>M^{\prime}$.

So, reducing to a subset of $B$ if necessary, we find that, for our chosen $n$ and $x$, the set $B \cap T^{-q_{n}^{k}} B$ takes up at least a fixed proportion of $Q_{i}^{n}(x)$; from (10) we have that for $n$ sufficiently large $\left\{y:\left|f_{q_{n}^{k}}(y)\right| \in \mathcal{K}\right\}$ also takes up at least a fixed proportion of $Q_{i}^{n}(x)$. Since these proportions add up to more than 1 , the two sets must intersect for $n=n_{j}>Y^{\prime}=\max \left\{M^{\prime}, N_{1}(0)\right\}$.

This shows that there is an $M$ with $B \cap T^{-m} B \cap\left\{y:\left|f_{m}(y)\right| \in \mathcal{K}\right\} \neq \emptyset$. Thus we have a contradiction, and the lemma is proved.

2.2.13 Theorem: Let $\int f d \mu=0, f^{(k-2)}$ be continuous with zero integral, $f^{(k-1)}$ be piecewise continuous also with zero integral and $f^{(k)}$ Riemann integrable with $\int f^{(k)} d \mu \neq 0$. Then the skew-product $T_{f}$ is ergodic for all $\alpha \in \mathcal{S}_{k}$.

\section{Proof:}

Since the only closed additive subgroups of $\mathbf{R}$ are: $\lambda \mathbf{Z}$ for $\lambda \geq 0$, or $\mathbf{R}$ itself, then the above Lemma and 1.1.4 demonstrate that the only possible remaining choice for the essential values is $E(f)=\mathbf{R}$. By 1.4.2 this shows that the skew-product $T_{f}$ is ergodic.

2.2.14 Remark: For the case where $k=1$, we note that $\mathcal{S}_{1}=\mathbf{R} \backslash \mathbf{Q}$, and so we obtain the result from $[\mathrm{P}]$. 


\section{THE ADDING MACHINE}

\subsection{Introduction}

In this Chapter we study skew product extensions of the adding machine transformation on $\mathbf{T}$ where the extension is defined by certain classes of real-valued functions. In the first case we study the class of functions which are piecewise continuous, have zero integral and have a derivative which is Riemann integrable with non-zero integral. We study this class of functions and show that their properties ensure that the skew-product is ergodic. We then note that the method used in Chapter 2, will not work for $k>1$.

Finally we study the class of functions which have zero integral and have a derivative of bounded variation, we show that these properties guarantee that these functions define cocycles which are always coboundaries for a certain class of adding machine.

\subsection{Conditions for ergodicity}

Here we proceed as in $[\mathrm{P}]$ for the adding machine:

3.2.1 Convention: Throughout this section we shall suppose that $f$ is piecewise continuous with zero integral, and has a derivative which is Riemann integrable with non-zero integral.

Let $f$ have discontinuities at $0 \leq \omega_{1}<\ldots<\omega_{N}<1$ where the ordering comes from the ordering of $\mathbf{T}$ by argument as in Chapter 2.

Let $d_{r}=f\left(\omega_{r}^{+}\right)-f\left(\omega_{r}^{-}\right)$for $r=1, \ldots, N$, where $f\left(\omega_{r}^{+}\right)$and $f\left(\omega_{r}^{-}\right)$are the limits of $f$ at $\omega_{r}$ as $x$ approaches from less or greater argument. We suppose without loss of generality that $\sum_{r=1}^{N} d_{r}>0$.

We define $P_{n}$ to be the partition of $\mathbf{T}$ into $p(n)$ intervals (n-cylinders), each of length $\frac{1}{p(n)}$, defined by the first $p(n)-1$ points on the orbit of 0 under $T$. The subscript of a cylinder denotes the $P_{n}$ to which it belongs. 
3.2.2 Lemma: For all $n$ and all cylinders $Q_{n}^{i} \in P_{n}, i=1, \ldots, p(n)$ there is a sub-interval (union of sub-cylinders) $J_{n}^{i} \subset Q_{n}^{i}$ on which $f_{p(n)}$ is continuous, and which satisfies:

$$
\frac{\mu\left(J_{n}^{i}\right)}{\mu\left(Q_{n}^{i}\right)} \geq \frac{1}{N}
$$

\section{Proof:}

Clearly, for all $n$ the discontinuities of $f_{p(n)}$ occur at the points $T^{s} \omega_{r}$ for $s=0, \ldots, p(n)-1$ and $r=1, \ldots, N$. In any cylinder $Q_{n}^{i} \in P_{n}$ there are, therefore, at most $N$ discontinuities of $f_{p(n)}$, and hence the result follows.

3.2.3 Lemma: There are positive constants $K_{1}, K_{2}$ and an integer $N_{1}$ such that for $n \geq N_{1}$ we have that

$$
n . K_{1}<f_{n}^{\prime}(x)<n . K_{2}
$$

for $\mu$-a.e. $x \in \mathbf{T}$.

\section{Proof:}

From the definition of $f$, and using Weyl's Theorem 1.5.3, we have that

$$
\frac{1}{n} \sum_{i=0}^{n-1} f^{\prime} \circ T^{i}(x) \rightarrow \int f^{\prime} d \mu
$$

uniformly for $\mu$-a.e. $x \in \mathbf{T}$. Given this, there is a positive integer $N_{1}$, such that for $n>N_{1}$ we obtain

$$
\left|\frac{f_{n}^{\prime}(x)}{n}-\int f^{\prime} d \mu\right|<\frac{1}{2} \int f^{\prime} d \mu,
$$

for $\mu$-a.e. $x \in \mathbf{T}$. So

$$
K_{1}<\frac{f_{n}^{\prime}(x)}{n}<K_{2}
$$

for $\mu$-a.e. $x \in \mathbf{T}$, where $K_{1}=\frac{1}{2} \int f^{\prime} d \mu$, and $K_{2}=\frac{3}{2} \int f^{\prime} d \mu$. 
3.2.4 Lemma: There exist strictly positive constants $H, F$ and an integer $N_{1}$ with the following properties: Within each cylinder $Q_{n}^{i}$ of $P_{n}$ for $i=1, \ldots, p(n)$, there is a sub-interval $J_{n}^{i}$ such that for $n>N_{1}$,

(i) $f_{p(n)}$ moves through a height greater than $H$ on $J_{n}^{i}$,

(ii) for any interval $[x, y]=I_{n}^{i} \subseteq f_{p(n)}\left(J_{n}^{i}\right)$, we have that

$$
\frac{\mu\left(f_{p(n)}^{-1}\left(I_{n}^{i}\right) \cap J_{n}^{i}\right)}{\mu\left(Q_{n}^{i}\right)}>F|y-x|
$$

\section{Proof:}

By Lemma 3.2.2 we may consider the cylinder $J_{n}^{i}=\left[a_{n}^{i}, b_{n}^{i}\right) \subset Q_{n}^{i}$ on which $f_{p(n)}$ is continuous and which satisfies

$$
p(n) \cdot \mu\left(J_{n}^{i}\right)=\frac{\mu\left(J_{n}^{i}\right)}{\mu\left(Q_{n}^{i}\right)} \geq \frac{1}{N} .
$$

Then from Lemma 3.2.3, since $p(n)>n$ for all $n$, we have for $n>N_{1}$, that

$$
K_{1} \cdot p(n)<f_{p(n)}^{\prime}(x)<K_{2} \cdot p(n),
$$

for $\mu$-a.e. $x \in \mathbf{T}$. Now we choose $H=\frac{K_{1}}{2 N}$, then for $n>N_{1}$ the height, $h$ moved through by the cocycle on $J_{n}^{i}$ is given by

$$
h=f_{p(n)}\left(b_{n}^{i}\right)-f_{p(n)}\left(a_{n}^{i}\right)=\int_{a_{n}^{i}}^{b_{n}^{i}} f_{p(n)}^{\prime} d \mu .
$$

In order to obtain a lower bound for this height, we use (15) and (16) above to give us

$$
h>K_{1} \cdot \mu\left(J_{n}^{i}\right) \cdot p(n) \geq \frac{K_{1}}{N} .
$$

This proves i).

For ii) define $m=f_{p(n)}^{-1}(x) \cap J_{n}^{i}$ and $p=f_{p(n)}^{-1}(y) \cap J_{n}^{i}$ then, since $f_{p(n)}$ is strictly increasing for $n>N_{1}$, we have that $f_{p(n)}^{-1}\left(I_{n}^{i}\right) \cap J_{n}^{i}=[m, p)$, and 


$$
y-x=\int_{m}^{p} f_{p(n)}^{\prime} d \mu .
$$

So, from (16) above we have, for $n>N_{1}$ that $p-m>\frac{y-x}{K_{2} \cdot p(n)}$. Hence we have that

$$
\frac{p-m}{\mu\left(Q_{n}^{i}\right)}>\frac{y-x}{K_{2}}
$$

and so

$$
\frac{\mu\left(f_{p(n)}^{-1}\left(I_{n}^{i}\right) \cap J_{n}^{i}\right)}{\mu\left(Q_{n}^{i}\right)}>\frac{y-x}{K_{2}}
$$

for $n>N_{1}$. Putting $F=\frac{1}{K_{2}}$, we note that $F$ is strictly positive and independent of $i$, which completes the proof of the second assertion.

\subsubsection{Lemma: $E(f) \neq \lambda \mathbf{Z}$ for any $\lambda \geq 0$.}

\section{Proof:}

Suppose that $E(f)=\lambda \mathbf{Z}$ where $\lambda \geq 0$. Since, by hypothesis $f$ has bounded variation, applying the Denjoy-Koksma inequality 1.5.1 gives us a $c>0$ such that $\left|f_{p(n)}(x)\right|<c$ for all $n$, and all $x \in \mathbf{T}$. Let $v$ be the greatest integer such that $v \lambda<c$. We choose

$$
0<\epsilon<\frac{H}{4(2 v+1)}
$$

where $H$ is the number obtained in Lemma 3.2.4i). Consider the compact set

$$
\mathcal{K}=(-\epsilon, c] \backslash \bigcup_{i=0}^{v}(i \lambda-\epsilon, i \lambda+\epsilon)
$$

This is the interval $(-\epsilon, c]$ without sub-intervals of width $2 \epsilon$ about each $i \lambda \in E(f)$, for $i=0, \ldots, v$. Clearly $E(f) \cap \mathcal{K}=\emptyset$, so applying Proposition 
1.1.6 we obtain a Borel set $B \in \Omega$ with $\mu(B)>0$ such that for all $m \in \mathbf{Z}$, $B \cap T^{-m} B \cap\left\{y:\left|f_{m}(y)\right| \in \mathcal{K}\right\}=\emptyset$.

In Lemma 3.2.4i) we showed that for all $n>N_{1}$, every cylinder $Q_{n}^{i}$ in $P_{n}$ contains a sub-interval $J_{n}^{i}$ on which $\left|f_{p(n)}\right|$ rises continuously through a height greater than $\frac{1}{2} H$. We claim that for $n$ sufficiently large, and for every cylinder $Q_{n}^{i}$, the ratio of the Lebesgue measure of $Q_{n}^{i}$ to that of the set $Q_{n}^{i} \cap\left\{y:\left|f_{p(n)}(y)\right| \in \mathcal{K}\right\}$ is greater than a fixed positive number.

Applying Lemma 3.2.4ii) to the interval(s) within $\mathcal{K}$ of total length at least $\frac{1}{2} H-(2 v+1) \epsilon>\frac{1}{4} H$, through which the graph of $\left|f_{p(n)}\right|$ must pass, gives us a strictly positive constant $F$ independent of $i$, such that, for $n>N_{1}$,

$$
\frac{\mu\left(J_{n}^{i} \cap\left\{y:\left|f_{p(n)}(y)\right| \in \mathcal{K}\right\}\right)}{\mu\left(Q_{n}^{i}\right)}>F \frac{H}{4} .
$$

So, by monotonicity, for $n>N_{1}$ there exists a strictly positive constant $W$, independent of $i$ such that

$$
\frac{\mu\left(Q_{n}^{i} \cap\left\{y:\left|f_{p(n)}(y)\right| \in \mathcal{K}\right\}\right)}{\mu\left(Q_{n}^{i}\right)}>W .
$$

This proves our claim.

Now, applying the Lebesgue Density Theorem, given any $x \in B \in \Omega$ with $\mu(B)>0$, and an $\epsilon=\frac{1}{2} W>0$, there is an $N_{2}$ such that for $n>N_{2}$

$$
\frac{\mu\left(Q_{n}^{i}(x) \cap B\right)}{\mu\left(Q_{n}^{i}(x)\right)}>1-\frac{1}{2} W .
$$

where $Q_{n}^{i}(x)$ is the cylinder in $P_{n}$ which contains $x$. Also, since we have that $T^{p(n)} Q_{n}^{i}(x)=Q_{n}^{i}(x)$ and the measure $\mu$ is $T$-invariant, we have that

$$
\frac{\mu\left(Q_{n}^{i}(x) \cap T^{-p(n)} B \cap B\right)}{\mu\left(Q_{n}^{i}(x)\right)}>1-W .
$$

So, for $n>\max \left\{N_{1}, N_{2}\right\}$, we have that the set $B \cap T^{-p(n)} B$ takes up at least a fixed proportion of $Q_{n}^{i}(x)$; from (17) above we also have that $\left\{y:\left|f_{p(n)}(y)\right| \in \mathcal{K}\right\}$ takes up at least a fixed proportion of $Q_{n}^{i}(x)$, for all $i$. Since these proportions add up to more than 1 , the two sets must intersect for this $n$.

This shows that there is an $m$ with $B \cap T^{-m} B \cap\left\{y:\left|f_{m}(y)\right| \in \mathcal{K}\right\} \neq \emptyset$. Thus we have a contradiction, and the lemma is proved. 
3.2.6 Theorem: Let $f$ be piecewise absolutely continuous, with $\int f d \mu=$ $0, f^{\prime}$ Riemann integrable and $\int f^{\prime} d \mu \neq 0$. Then the skew product $T_{f}$ is ergodic.

\section{Proof:}

Since the only closed additive subgroups of $\mathbf{R}$ are: $\lambda \mathbf{Z}$ for $\lambda \geq 0$, or $\mathbf{R}$ itself, the above Lemma and 1.1.4 demonstrate that the only possible remaining choice for the essential values is $E(f)=\mathbf{R}$. By Theorem 1.4.2 this shows that the skew product $T_{f}$ is ergodic.

3.2.7 Remark: We note that the arguments above and in Chapter 2 involve properties of the sequences of integers $p(n), q_{n}$ respectively associated to the map $T$. Hence it seems that we may obtain similar results to those given in Chapter 2 for the adding machine transformation. However in Chapter 2, for the class of functions defined for $k>1$ in 2.2.1, we must use the 'good approximation' conditions of our chosen irrational numbers to ensure that as we iterate our partition intervals around by $T^{q_{n}}$, the discontinuities do not spread evenly over the interval. For the adding machine, this behaviour does not occur, and so the method will not be applicable.

\subsection{Coboundaries for the adding machine}

Now we study a different class of functions, which define cocycles which have extremely different cohomological properties to those studied in the previous subsection.

3.3.1 Convention: In this section we study the class of functions with zero integral and have a derivative which has bounded variation. We also assume that the associated integers $p(n)$ (see 1.3) of our adding machine transformation satisfy

$$
\sum_{n=1}^{\infty} \frac{q_{n+1}}{p(n)}<\infty .
$$


3.3.2 Lemma: Suppose $f$ is as above, then there is a positive constant $K$ such that, for all $n$ and all $x \in \mathbf{T}$

$$
\left|f_{p(n)}(x)\right|<\frac{K}{p(n)}
$$

\section{Proof:}

Since $f^{\prime}$ has bounded variation and $\int f^{\prime} d \mu=0$, the Denjoy-Koksma inequality 1.5.1 gives us that

$$
\left|f_{p(n)}^{\prime}(x)\right|<c^{\prime}
$$

for some positive constant $c^{\prime}=\operatorname{var} f^{\prime}$, all $x \in \mathbf{T}$ and all $n$. Since $\int f d \mu=0$, we also note that for all $n$, we have that

$$
\int_{B_{n}} f_{p(n)} d \mu=0
$$

on any $n$-cylinder, $B_{n} \subset \mathbf{T}$. By hypothesis $f$ is continuous, so we may assume that there is a positive constant $c$ such that $|f(x)|<c$ for all $x \in \mathbf{T}$.

Let $K=\max \left\{c, 3 c^{\prime}\right\}$, then suppose without loss of generality, that there is an $x \in \mathbf{T}$ and an $n$ such that $f_{p(n)}(x) \geq \frac{K}{p(n)}$. We now show that if this is the case, then there is an $n$-cylinder on which the integral of $f_{p(n)}$ cannot be zero.

From (20) the slope of $f_{p(n)}$ is bounded for all $n$, hence we may calculate the proportion of the $n$-cylinder to which $x$ belongs on which $f_{p(n)}$ is positive. Let $d_{1}, d_{2}$ denote the distances to the right and left of $x$ respectively, when $f_{p(n)}$ next crosses the $x$-axis. Since $f$ is absolutely continuous we have that

$$
f_{p(n)}(x)=\int_{x}^{x+d_{1}} f_{p(n)}^{\prime} d \mu,
$$

and so, by assumption and (20)

$$
d_{1}>\frac{K}{c^{\prime}} \cdot \frac{1}{p(n)} \geq 3 \cdot \frac{1}{p(n)} .
$$

We get a similar inequality for $d_{2}$. This implies that the function $f_{p(n)}$ cannot return to zero inside the cylinder. Therefore we cannot have $\int f_{p(n)} d \mu=0$ for this cylinder, this contradicts (21), and so the result follows. 
3.3.3 Theorem: Suppose that $f: \mathbf{T} \rightarrow \mathbf{R}$ is such that $\int f d \mu=0$, with $f^{\prime}$ of bounded variation, then for almost all $x \in \mathbf{T}$, we have that

$$
\sup _{n}\left|f_{n}(x)\right|<\infty
$$

and hence from Theorem 1.5.2, $f$ defines a coboundary whose cobounding function is $L^{\infty}$.

\section{Proof:}

Any $n \in \mathbf{N}$ may be written uniquely as

$$
n=\sum_{i=0}^{s(n)} n_{i} p(i) \text { where } n_{i} \in\left\{0, \ldots, q_{i+1}-1\right\}
$$

for some positive integer $s(n)$. Thus for all $x \in \mathbf{T}$, we may write $f_{n}(x)$ as

$$
f_{n}(x)=f_{p(0) n_{0}}(x)+f_{p(1) n_{1}}\left(T^{n_{0}} x\right)+\ldots+f_{p(s(n)) n_{s(n)}}\left(T^{\sum_{i=0}^{s(n)-1} p(i) n_{i}} x\right),
$$

by the cocycle relation. Hence by the cocycle relation (1) again, and the triangle inequality we have that

$$
\left|f_{n}(x)\right| \leq\left|n_{0} f(x)\right|+\ldots+\left|n_{s(n)} f_{p(s(n))}\left(T^{\sum_{i=0}^{s(n)-1} p(i) n_{i}} x\right)\right| .
$$

Using (19) we have that

$$
\left|f_{n}(x)\right| \leq n_{0} \cdot K+\ldots+n_{s(n)} \cdot \frac{K}{p(s(n))}<K \cdot \sum_{i=0}^{s(n)} \frac{q_{i+1}}{p(i)}<\infty
$$

since $n_{i}<q_{i+1}$ for all $i$, and using our hypothesis (18). Hence the result follows. 


\section{References}

[AS] G. Atkinson and K. Schmidt,

Functions whose sum of translates remains bounded, Preprint: Warwick 1977.

[B] L. Baggett, On functions that are trivial cocycles for a set of irrationals, Proc. Amer. Math. Soc. 104 (1988) 1211-1215.

[BM] L. Baggett and K. Merrill, Equivalence of cocycles under an irrational rotation, Michigan Math. J. 33 (1986) 221-229.

[C] P. Cohn, Measure theory, Birkhaüser Boston (1980).

[Ca] J. W. S. Cassels, An introduction to Diophantine approximation, Cambridge University Press (1957).

[D] A. Denjoy, Les trajectories á la surface du tore, C.R. Acad. Sci. Paris, 223 (1946), 5-8.

[H1] P. Hellekalek, Regularities in the distribution of special sequences, J. Number Theory, 18 (1984), 41-55.

[H2] P. Hellekalek, Ergodicity of a class of cylinder flows related to irregularities of distribution, Compositio Mathematica, 61 (1987), 129-136.

[He] M. Herman, Sur la conjugaison différentiable des difféomorphismes du cercle a des rotations, DSc. Thesis, Orsay (1976).

[HL1] P. Hellekalek and G. Larcher, On the ergodicity of a class of skew products, Israel J. Mathematics, 54 (1986), 301-306. 
[HL2] P. Hellekalek and G. Larcher,

On Weyl sums and skew products over irrational rotations, To appear Theoret. Computer Sci., 65 (1989).

[HL3] P. Hellekalek and G. Larcher, Functions with bounded remainder, Preprint: Salzburg 1987.

[HR] E. Hewitt and K. Ross, Abstract Harmonic Analysis, Volume I. Springer Verlag, Heidelberg New York Berlin (1963).

[HW] G. Hardy and E. Wright, An introduction to the theory of numbers, Fourth edition, Oxford University Press (1959).

[K] Y. Katznelson, Sigma-finite invariant measures for smooth mappings of the circle, J. D'Analyse, 33 (1977), 1-18.

[KS] K. Schmidt, Cocycles of ergodic transformation groups, Lecture Notes in Mathematics, Vol. 1, MacMillan Co. of India, New Dehli, 1977.

[O] I. Oren, Ergodicity of cylinder flows arising from irregularities of distribution, Israel J. Mathematics, 44 (1983), 127-138.

[P] D. Pask, Skew products over the irrational rotation, Preprint: Warwick 1989.

[Pe1] K. Peterson, On a series of cosecants related to a problem in ergodic theory, Compositio Mathematica, 26 (1973), 313-317.

[Pe2] K. Peterson, Ergodic theory, Cambridge University Press (1983).

[R] W. Rudin, Fourier Analysis on groups, Interscience, Wiley (1967). 